ISSN 1112-9867

http://www.jfas.info

\title{
DETECTION OF STATIC ECCENTRICITY FAULT IN PSH INDUCTION MOTOR BY USING EXTERNAL MAGNETIC FLUX DENSITY
}

\author{
N. Halem ${ }^{1,2, *}$, S. E. Zouzou ${ }^{2}$, H. Ghodbane ${ }^{3}$ \\ ${ }^{1}$ University of El Oued, Faculty of Technology, Department of Electrical Engineering, \\ PO Box 789, 39000, El Oued, Algeria \\ ${ }^{2}$ University of Biskra, LGEB Laboratory, PO Box 145, 07000, Biskra, Algeria \\ ${ }^{3}$ University of Biskra, LMSE Laboratory, PO Box 145, 07000, Biskra, Algeria
}

Received: 29 May 2016 / Accepted: 25 August 2016 / Published online: 01 September 2016

\begin{abstract}
The objective of this paper is to make a clear and thorough investigation about the effectiveness of Motor Current Signature Analysis (MCSA) for detecting default indexes of static eccentricity in (Principal Slot Harmonics) PSH induction motor. The obtained results using traditional technique (MCSA) are compared with spectrums of external magnetic flux density extracted from healthy and faulty motors. The work is carried out with transient finite element method (FEM) simulations considering motor operation under nominal mechanical load, the notching nature of stator and rotor and the non-linearity behavior of ferromagnetic material are taking into account.
\end{abstract}

Keywords: induction motor; static eccentricity; FEM, PSH; MCSA; magnetic flux density.

Author Correspondence, e-mail: noura.halem@yahoo.fr

doi: http://dx.doi.org/10.4314/jfas.v8i3.10

\section{INTRODUCTION}

Le stress mécanique affectant les moteurs à induction est, dans la plupart des cas causé par un déséquilibre dans l'attraction magnétique. Cette situation génère au fil du temps des ruptures, 
des frottements finissant par entrainer des pannes majeures dans le moteur.

Le stress mécanique est induit par le défaut d'excentricité. Ce défaut se présente sous deux formes couramment jumelées en permanence: L'excentricité statique et l'excentricité dynamique [1-5].

Il est admis que le défaut d'excentricité statique est généré lors du processus de fabrication et d'usinage du moteur triphasé à induction. Ce défaut initial est perçu par les concepteurs comme tolérable au seuil de $10 \%$ (essentiellement pour les moteurs à induction de grande puissance). Il est par ailleurs admis qu'il faut redouter l'effet d'entrainement des défauts induits dans le temps et cela quelque soit le pourcentage initial du défaut d'excentricité statique. Nandi, dans sa recherche stipule que le défaut dynamique provient du défaut d'excentricité statique [6-10]. En fait tout se passe comme ci le défaut d'excentricité statique évolue en se complexifiant entrainant le défaut d'excentricité dynamique. Ainsi, se justifie le diagnostique du défaut d'excentricité statique sans tenir compte du seuil toléré évoqué plus haut. Cette idée constitue l'objectif de cet article où nous essayerons de présenter l'importance de diagnostiquer le défaut d'excentricité statique à des seuils inferieurs à $10 \%$.

La résolution de cette problématique passe par la réponse aux trois problèmes suivants:

- La première difficulté majeure est de distinguer uniquement le défaut d'excentricité statique, car expérimentalement ce défaut est lié à des seuils divers au défaut d'excentricité dynamique, ceci impose le passage à priori par la simulation [9].

- Le deuxième problème provient de la modélisation qui est répartit en deux catégories: les méthodes analytiques et les méthodes numériques. L'approche analytique qui est la plus abordable par sa simplicité et sa célérité, offre plusieurs modèles dont la plupart sont à hypothèses simplificatrices. Elle est incapable d'intégrer le comportement magnétique et la géométrie du moteur. En plus cette approche à montrer ses limites de détection de défauts d'excentricité statique de faibles pourcentages (Les références dans cette rubrique abordent des taux de $30 \%$ et plus).

- La troisième limite est lié aux méthodes de diagnostique de défaut d'excentricité statique, plusieurs techniques existent et toutes se basent sur l'analyse des signaux du moteur (courant statorique [11-13], couple électromagnétique [14], densité du flux magnétique 
[15-22], vibrations....). La technique la plus fréquemment utilisée est l'analyse spectrale du courant statorique, choisie pour sa simplicité. Cette méthode auparavant très en vogue montre des limites quant à la détection des défauts d'excentricité statique seul. Elle est inefficace en présence de deux harmoniques principaux d'encochage (Principal Slot Harmonics) PSH.

$\mathrm{Au}$ regard de ces imperfections, nous tenterons dans ce travail de trouver des palliatifs à ces limites par l'utilisation d'un modèle d'éléments finis pour la modélisation du moteur à induction sain et celle présentant un défaut d'excentricité statique. Cette méthode se distingue par des avantages certains vis à vis des méthodes analytiques; elle tient compte de la saturation magnétique des parties ferromagnétiques du moteur, et de l'effet d'encochage.

Les spectres du courant statorique et de l'induction magnétique à l'extérieur du moteur seront analysés à partir des modèles simulés (moteur sain et avec défaut d'excentricité statique) dans le but d'investiguer l'existence des indices de défaut. Le moteur à étudier dans cet article présente les deux PSH.

\section{MODÈLE ÉLÉMENT FINIS DU MOTEUR À INDUCTION TRIPHASÉ À CAGE}

La méthode d'éléments finis pas à pas dans le temps connue sous le générique TSFEM (Time Stepping Finite Element Method), est utilisée pour la modélisation et l'étude du moteur à induction sain et défectueux. Le but recherché est d'obtenir un espace d'harmonique permettant d'appliquer la technique d'analyse des signaux en vue de diagnostic des différents défauts dans le moteur à induction. Ce type de modélisation est très coûteux en temps de calculs, mais il donne une bonne vision de la dynamique du moteur sain et lors de l'apparition d'un défaut. Cette caractéristique est à l'origine de l'engouement pour cette méthode en témoigne le nombre pléthorique des travaux notamment pour le diagnostic des moteurs à induction.

Le modèle éléments finis correspond à notre moteur à induction de $1.1 \mathrm{~kW}, 230 \mathrm{~V}, 50 \mathrm{~Hz}$ et 4 pôles est représentée par la figure 1 . 


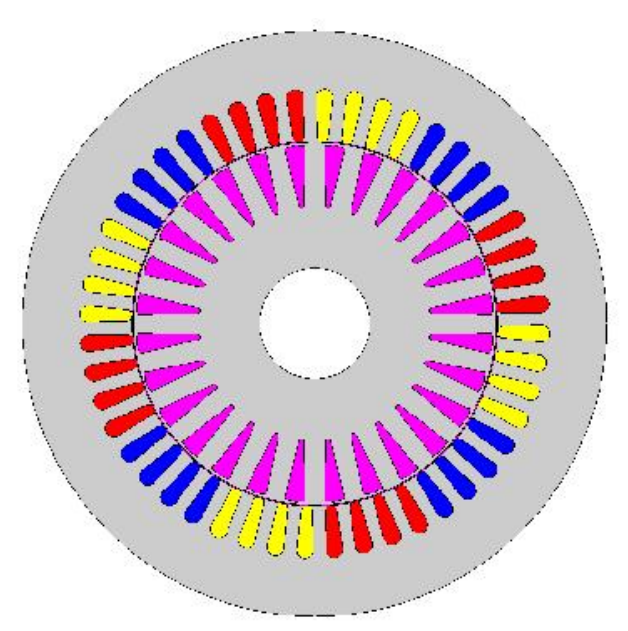

Fig.1. Structure du moteur étudié

Table 1. Caractéristiques du moteur

\begin{tabular}{ll}
\hline Nombre de pôles & 4 \\
Nombre de phases & 3 \\
Puissance $(\mathrm{kW})$ & 1.1 \\
Tension (V) & 230 \\
Fréquence (Hz) & 50 \\
Vitesse (rpm) & 1425 \\
Nombre des encoches statoriques & 48 \\
Nombre des encoches rotoriques & 28 \\
\hline
\end{tabular}

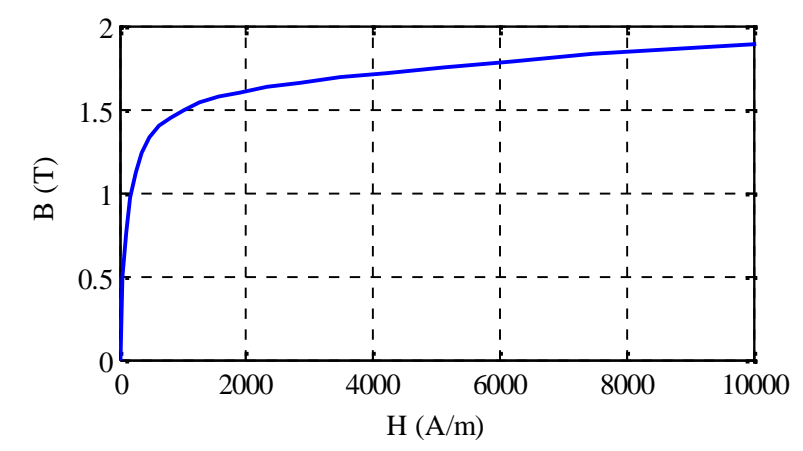

Fig.2. Caractéristiques $\mathrm{B}(\mathrm{H})$ des parties ferromagnétiques

L'application du modèle est du type magnétique transitoire avec couplage circuit qui permet l'étude des phénomènes créés par un champ magnétique variable dans le temps. Le champ magnétique est lié à la présence de courants électriques variables. Cette application permet la 
prise en compte des courants induits dans les régions conductrices (courants de Foucault). Elle permet également la prise en compte, dans ces régions conductrices, des effets de peau et des effets de proximité. La variable d'état du champ électromagnétique-le potentiel vecteur magnétique $A(x, y, z, t)$, satisfait les équations différentielles suivantes [23]:

$$
\begin{aligned}
& \operatorname{cur}[1 / \mu \cdot \operatorname{curl} A]+(\partial A / \partial t) / \rho=J_{s}(x, y, z, t) \\
& \operatorname{div} A=0
\end{aligned}
$$

Où $\mu$ représente la perméabilité magnétique du matériau, $\rho$ la résistivité du matériau et $J_{s}$ la densité de courant dans les encoches statoriques. Le terme $(\partial A / \partial t) / \rho$ représente la densité du courant induit qui est non nulle uniquement dans les régions correspondant aux conducteurs massifs des encoches rotoriques. Dans le modèle du champ considéré pour cette étude, la source de densité de courant a la forme $J_{s}\left[0,0, J_{s}(x, y, z, t)\right]$. En conséquence, le potentiel vecteur $A_{s}\left[0,0, A_{s}(x, y, z, t)\right]$ est orienté selon l'axe $\mathrm{Oz}$ et ne dépend pas de la coordonnée z. Dans ce cas la deuxième équation 1 est implicitement satisfaite.

\section{RÉSULTATS ET DISCUSSION}

\subsection{Analyse spectrale du courant statorique du moteur sain}

À partir du modèle magnétodynamique pas à pas dans le temps, on a obtenu le courant statorique du moteur. Le spectre du courant statorique du moteur sain en pleine charge est représenté sur la figure 3.

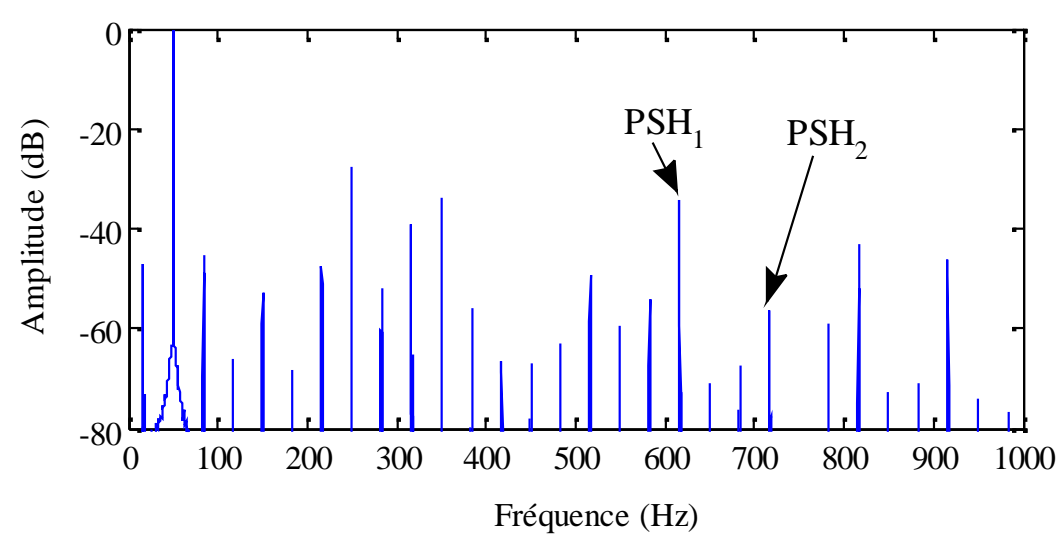

Fig.3. Spectre du courant statorique du moteur sain en pleine charge

D'après la figure 3 , les harmoniques prévus sont apparus dans le spectre du courant statorique 
pris du modèle non-linéaire, ces harmoniques sont classés comme suit [24-27]:

- Une série d'harmoniques dus à la saturation (Saturation Harmonics, Time Harmonics): qui sont localisées aux fréquences; $150 \mathrm{~Hz}, 450 \mathrm{~Hz}$, et $750 \mathrm{~Hz}$, ces fréquences sont tirées à partir de la formule:

$$
H_{s}=3 k f_{s}
$$

Avec k impair.

- Une série des harmoniques de la force magnétomotrice (fmm) (mmf harmonics): ces harmoniques sont les plus éminents dans le spectre du courant statorique du moteur à induction, leur présence est conforme à la définition de la fmm, ils résultent du courant qui traverse le bobinage statorique et donc c'est une conséquence de la nature discrète des enroulements statoriques. Les composantes fréquentielles de la fmm se situent aux fréquences: $50 \mathrm{~Hz}, 250 \mathrm{~Hz}, 350 \mathrm{~Hz}$, et $550 \mathrm{~Hz}$ sont donnés par la formule:

$$
H_{m}=(6 k \mp 1) f_{s}
$$

Avec $(k=\mp 1, \mp 2, \ldots)$

- Une série d'harmoniques d'encoches rotoriques RSHs (Rotor Slot Harmonics): leur existence ne dépend pas seulement de la fmm, la perméance du stator et du rotor et la denture de stator et de rotor, mais ils existent aussi dans le spectre du courant de statorique que pour un nombre combiné de pair de pôles et les barres rotoriques.

Généralement les fréquences des RSHs sont données par la formule suivante [27]:

$$
f_{H}=\left|\left(\lambda \mp\left(k R+n_{d}\right) \frac{(1-s)}{p}\right) f_{s}\right|
$$

$f_{s}$ : la fréquence du réseau d'alimentation.

$\lambda$ : le rang des harmoniques du temps $(\lambda=\mp 1, \mp 3, \mp 5, \ldots$.$) .$

$R$ : le nombre des barres rotoriques.

$s:$ le glissement.

$p$ : le nombre de pair de pôles. 
$k$ et $n_{d}$ : sont des coefficients liés à l'excentricité, $n_{d}=0$ c'est le cas d'excentricité statique, $n_{d}=1,2,3, \ldots$, c'est le cas d'excentricité dynamique $\left(n_{d}\right.$ est le rang des harmoniques d'excentricité). Pour le cas d'un moteur sain $k=0$ et $n_{d}=0$, alors on obtient les fréquences des harmoniques d'encoches rotoriques comme suit:

$$
\begin{gathered}
f_{R S H s}=\left|\left(\lambda \mp R \frac{(1-s)}{p}\right) f_{s}\right| \\
f_{r}=\frac{(1-s)}{p} f_{s} \\
f_{R S H s}=\left|\lambda f_{s} \mp R f_{r}\right|
\end{gathered}
$$

La relation qui relie le nombre de pair de pôles et le nombre des encoches rotoriques est assurée par la formule suivante [8-9]:

$$
R=2 p[3(m \pm q) \pm r] \pm k
$$

Avec: $\mathrm{R}$ est le nombre des encoches rotoriques, $m \pm q=0,1,2,3 \ldots, \quad r=0$ ou $1, \quad k=1$ ou 2 . On peut distinguer trois cas selon la valeur du k:

a) Pour $k=1$, la formule 4 sera bien satisfaisante pour observer les harmoniques du défaut d'excentricité; soit statique pure ou dynamique pure qui seront fortement présentés dans le spectre du courant statorique. Dans ce cas les deux PSH ne sont pas générés dans le spectre du courant statorique.

b) Pour $k=2$, les harmoniques caractérisant le défaut d'excentricité statique, ou dynamique, seront faiblement présentés dans le spectre du courant statorique et cela seulement pour de très faibles charges c'est à dire pour une vitesse qui vient presque à atteindre la vitesse de synchronisme.

c) Les PSH ne se révèlent dans le spectre du courant statorique, que si la formule suivante est satisfaite:

$$
R=2 p[3(m \pm q) \pm r]
$$


Le cas du moteur étudié qui possède 28 encoches rotoriques et 2 pair de pôles $p=2$, pour $m \pm q=2$ et $r=1$, on obtient:

$$
R=2(2)[3(2) \pm 1]=28
$$

La formule 9 est satisfaisante, et ainsi on a: $(R / p=28 / 2=14)$, et d'ailleurs les deux PSH sont clairement observés dans le spectre du courant statorique (figure 3). D'après la littérature [8-9], dans ce cas le moteur est appelé: PSH-Moteur (PSH-Motor).

Pour ce type de moteurs où les deux PSH existent dans le spectre du courant statorique, l'intérêt sera porté sur les harmoniques caractérisant le défaut d'excentricité statique seul ou dynamique seul absentés dans le spectre du courant statorique.

d) Exceptionnellement, pour le défaut d'excentricité mixte c'est à dire dans le cas où le moteur présente les deux types d'excentricité statique et dynamique, les composantes fréquentielles qui caractérisent ce défaut seront produites dans le spectre du courant statorique pour tous les types des moteurs à induction, et quel que soit la combinaison entre le nombre de pair de pôles et le nombre des barres rotoriques.

En effet, l'existence des PSH dans le spectre du courant statorique joue un rôle primordial dans le diagnostic des défauts du moteur à induction, particulièrement pour la détection des défauts d'excentricités statique et dynamique, et cela sera bien vérifié dans le paragraphe suivant.

$\mathrm{Si}$, par conséquent, le moteur à induction possède les deux $\mathrm{PSH}$, cela provoque la génération des autres composantes fréquentielles d'ordres supérieures $(3,5, \ldots)$. Ces composantes fréquentielles possèdent généralement des amplitudes importantes et cela contribuent fortement dans la procédure de diagnostic en contrôlant leurs amplitudes. D'après le spectre présenté dans la figure 3, on peut citer par exemple les harmoniques les plus importants d'ordre 3 et 5 caractérisés par les fréquences suivantes:

$\left|(\lambda \mp R(1-s) / p) f_{s}\right|=|(3 \mp 28(1-0.048) / 2) 50|=516.4 \mathrm{~Hz}$ et $816.4 \mathrm{~Hz}$.

$\left|(\lambda \mp R(1-s) / p) f_{s}\right|=|(5 \mp 28(1-0.048) / 2) 50|=416.4 \mathrm{~Hz}$ et $916.4 \mathrm{~Hz}$.

Il faut remarquer que dans la littérature ces harmoniques $(516.4 \mathrm{~Hz}, 816.4 \mathrm{~Hz}, 416.4 \mathrm{~Hz}$ et 
916.4 Hz) sont appelés: les harmoniques reliés à la saturation (saturation-related harmonic).

\subsection{Analyse spectrale du courant statorique et de l'induction magnétique à l'extérieur du moteur avec défaut d'excentricité statique}

L'analyse porte sur deux grandeurs du moteur à induction; le courant statorique et la densité du flux magnétique à l'extérieur du moteur. L'analyse du courant statorique à base de la technique de MCSA est considérée pour confirmer l'absence des indices de défaut d'excentricité statique.

Le but principal est la détection des signatures de défaut d'excentricité qui sont données par la formule (4) [87-89]:

Les indices de défaut d'excentricité qui se trouvent aux basses fréquences autour de la composante fondamentale $(50 \mathrm{~Hz})$, sont donnés par la formule suivante:

$$
f_{\text {exc }}=\left(f_{s} \mp k f_{r}\right)
$$

La modélisation par la méthode des éléments finis a permis de visualiser les grandeurs spatiales du moteur, on peut ici visualiser les lignes de flux magnétique au fonctionnement en pleine charge à l'extérieur et à l'intérieur du moteur aux différents pas de temps $0.001 \mathrm{~s}$, et $4 \mathrm{~s}$ pour les cas suivants : moteur sain, moteur avec $05 \%$ et $10 \%$ d'excentricité statique (figure 4 ). Les lignes de champ magnétique correspondant au moteur sain à diffèrent pas de temps sont toujours symétriques, lorsque le moteur à induction a une excentricité statique, les lignes d'équiflux sont très influencées par le défaut et elles sont distribuées d'une façon non uniforme.

Les figures 5 et 6 représentent respectivement les spectres du courant statorique du moteur sain et moteur avec défaut d"exentricité statique, aucune différence entre les deux spectres, seulement on peut noter des petites perturbations des amplitudes des harmoniques permanents.

Les figures 7 et 8 montrent respectivement les spectres de l'induction magnétique à l'extérieur du moteur sain et moteur avec défaut d'excentricité statique. En premier lieu, on peut noter la présence de tous les harmoniques permanents dans le spectre de la densité du flux magnétique, aussi la différence entre les deux spectres est très clair, alors, on peut facilement détecter l'apparition des nouvelles composantes fréquentielles dans le spectre du moteur avec défaut. 

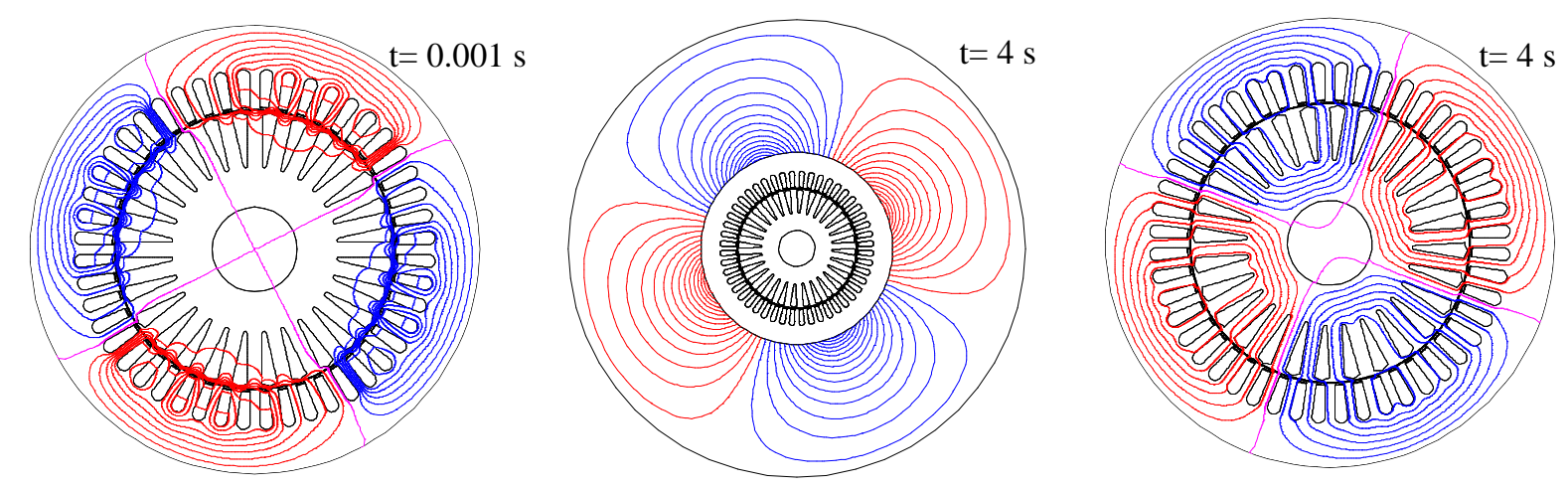

a) Moteur sain
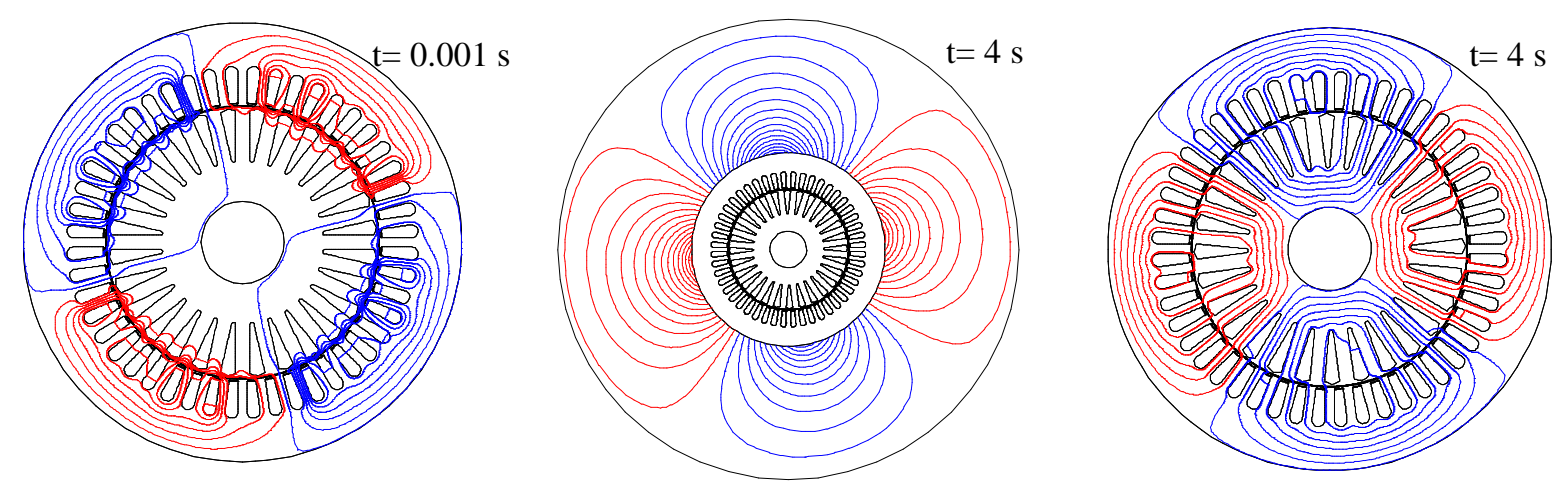

b) Moteur avec défaut ; 05\% excentricité statique
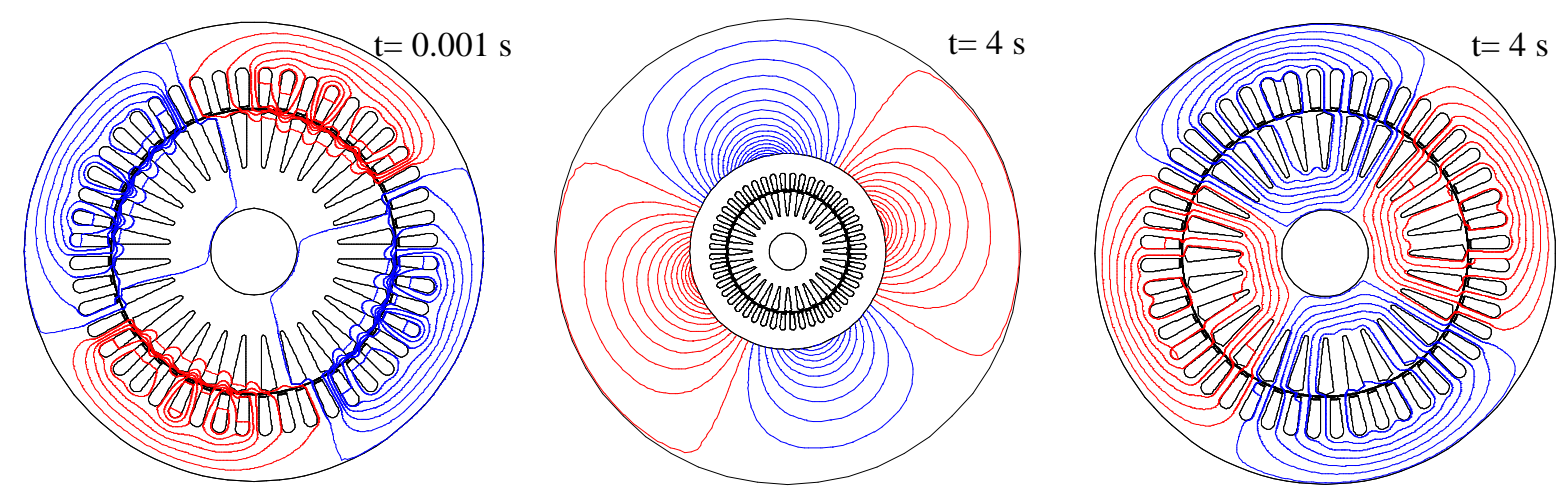

c) Moteur avec défaut ; $10 \%$ excentricité statique

Fig.4. Distribution des lignes de flux à l'intérieur et à l'extérieur du moteur sain et du moteur avec défaut d'excentricité statique (en pleine charge) 


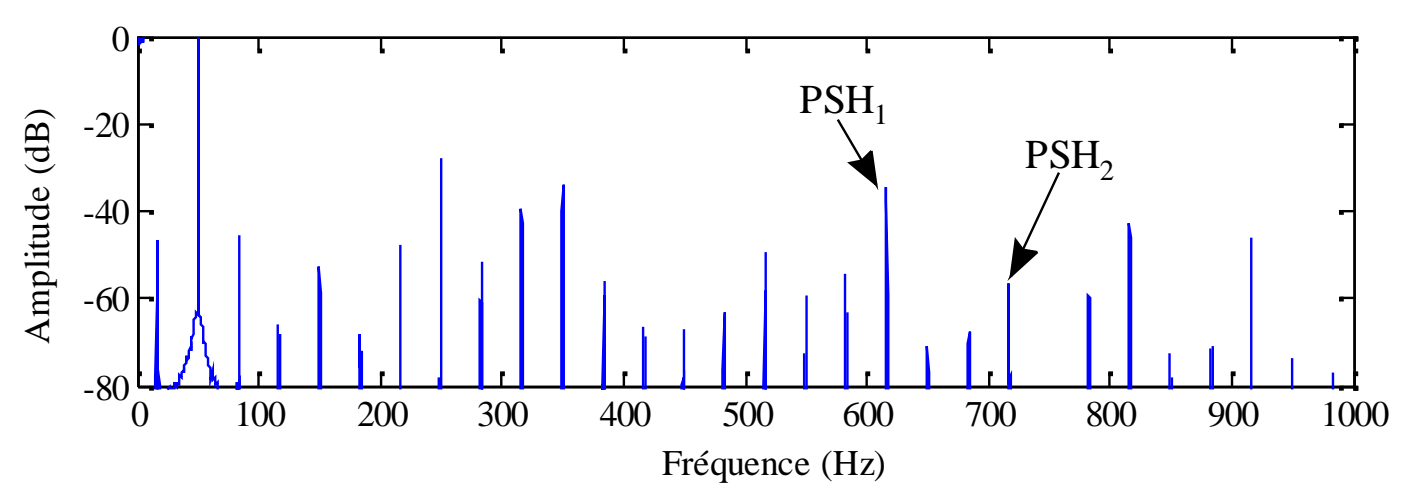

Fig.5. Spectre du courant statorique du moteur sain en pleine charge

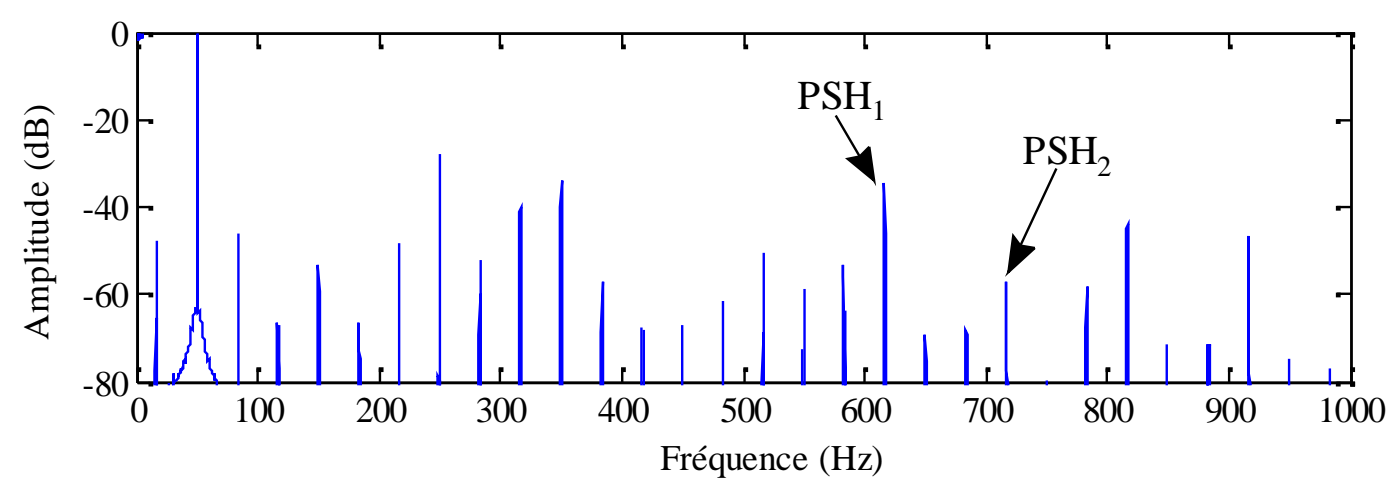

Fig.6. Spectre du courant statorique du moteur avec défaut d'excentricité en pleine charge

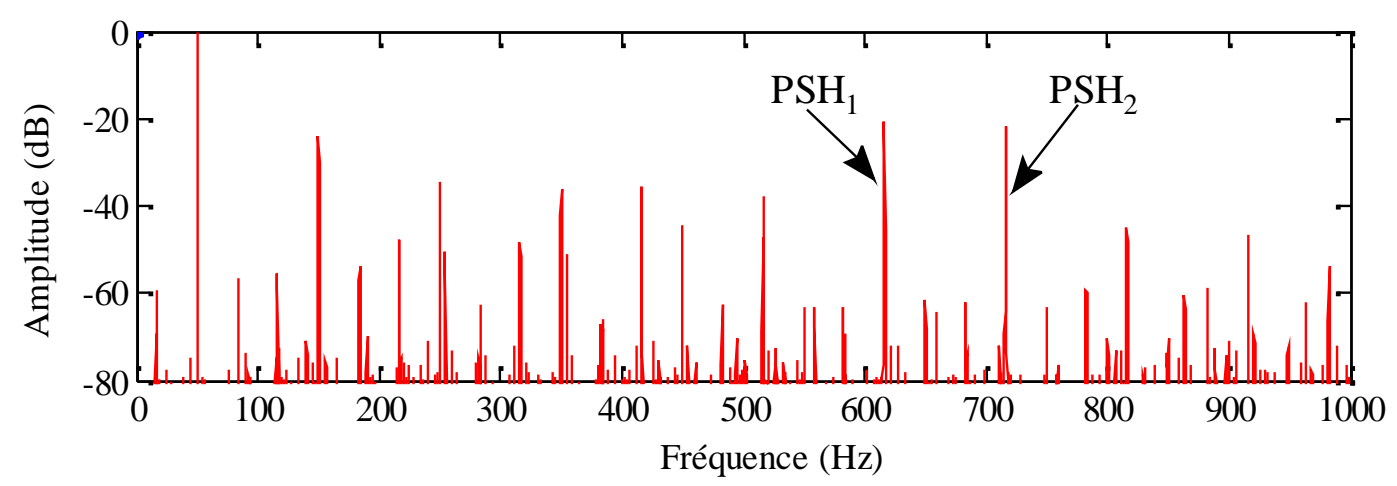

Fig.7. Spectre de l'induction magnétique à l'extérieur du moteur sain en pleine charge

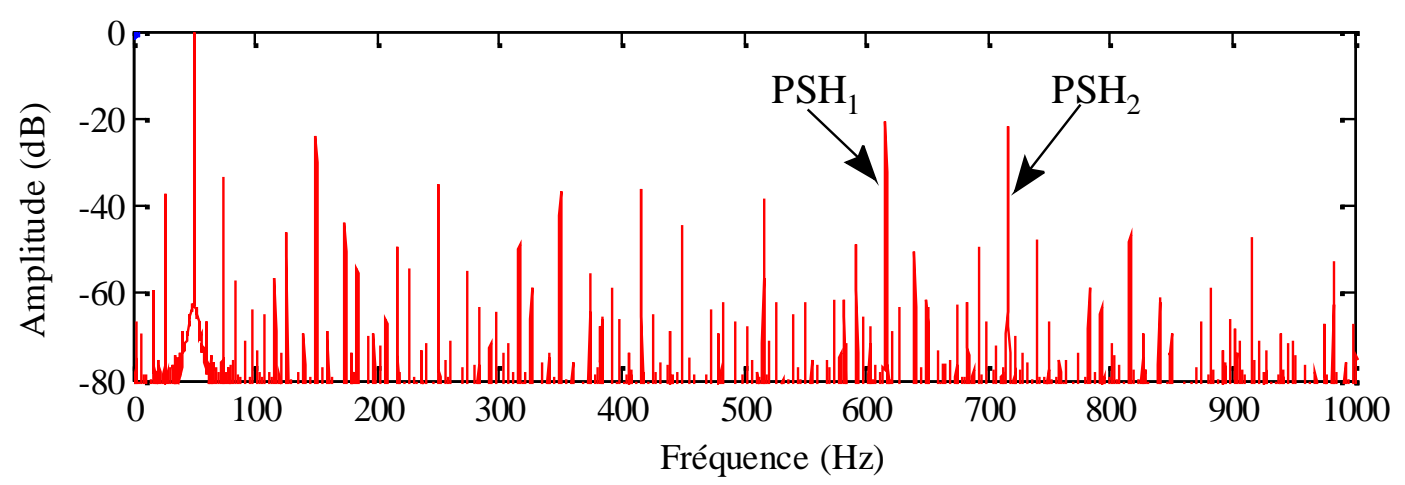

Fig.8. Spectre de l'induction magnétique à l'extérieur du moteur avec défaut d'excentricité en pleine charge 
La figure 9 représente les spectres du courant statorique et de l'induction magnétique à l'extérieur du moteur sain et moteur avec $05 \%$ et $10 \%$ d'excentricité statique. La simulation a été effectuée pour un moteur fonctionnant en pleine charge. D'après cette figure on peut retirer les remarques suivantes:

- Aucune signature de défaut n'est produite dans les spectres de courant statorique (figures 9.b et 9.c), seulement on relève de légères perturbations concernant les amplitudes de RSHs.

- Les signatures de défaut d'excentricité statique sont clairement apparentes dans les spectres de l'induction magnétique à l'extérieur du moteur (figures 9.b' et 9.c') pour les deux degrés de défaut, elles sont situées aux fréquences:

$$
f_{s} \mp f_{r}=(1 \mp(1-s) / p) f_{s}=(1 \mp(1-0.05) / 2) 50=26.19 \mathrm{~Hz} \text { et } 73.85 \mathrm{~Hz} \text {. }
$$

- En plus, les signatures $\left(f_{s} \mp f_{r}\right)$ autour de la composante fondamentale, avec une analyse minutieuse de spectres avec défaut, nous permet de noter des signatures spectrales liées au défaut d'excentricité statique:

Les signatures de défaut autour de la composante fondamentale de rang élevé, comme les fréquences:

$$
\left(f_{s} \mp 2 f_{r}\right)=\left(f_{s} \mp 2((1-s) / p) f_{s}\right)=(50 \mp 2((1-0.05) / 2) 50)=2.5 \mathrm{~Hz} \text { et } 97.5 \mathrm{~Hz}
$$

Les signatures de défaut d'excentricité autour de la troisième composante du courant (150 $\mathrm{Hz}$ ) sont:

$$
\left(3 f_{s} \mp f_{r}\right)=(3 \cdot 50 \mp((1-0.05) / 2) 50)=126.25 \mathrm{~Hz} \text { et } 173.75 \mathrm{~Hz}
$$

Les signatures de défaut d'excentricité autour de deux PSH sont:

$$
f_{s}-(R \mp 1)((1-s) / p) f_{s}=f_{s}-(R \mp 1) f_{r}=50-(28 \mp 1)((1-0.05) / 2) 50=591.25 \mathrm{~Hz} \text { et }
$$
$638.75 \mathrm{~Hz}$.

$f_{s}+(R \mp 1)((1-s) / p) f_{s}=f_{s}+(R \mp 1) f_{r}=50+(28 \mp 1)((1-0.05) / 2) 50=691.25 \mathrm{~Hz}$ et $738.75 \mathrm{~Hz}$. 


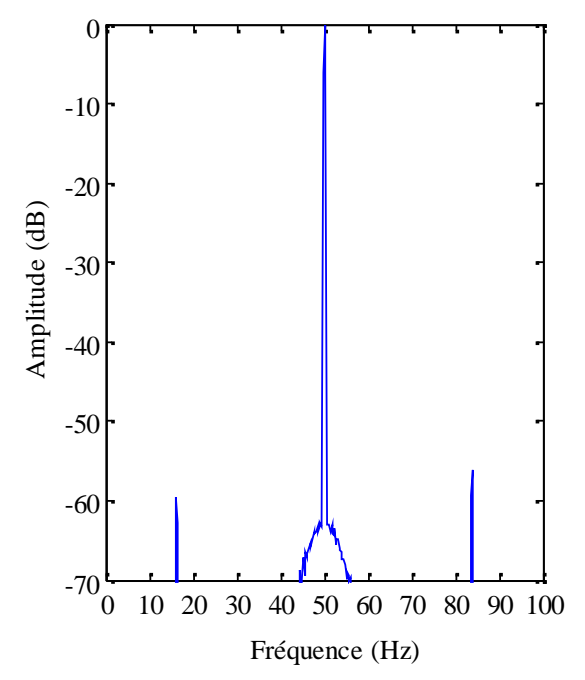

a) Courant statorique à l'état sain

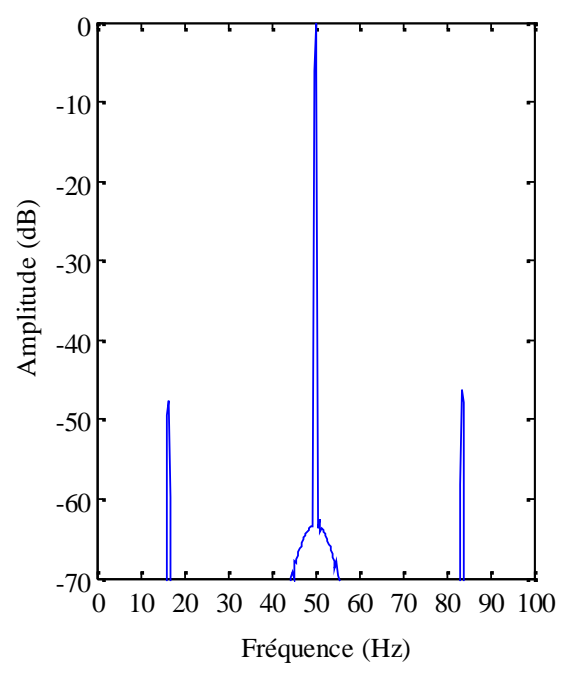

b) Courant statorique avec $05 \%$ de défaut

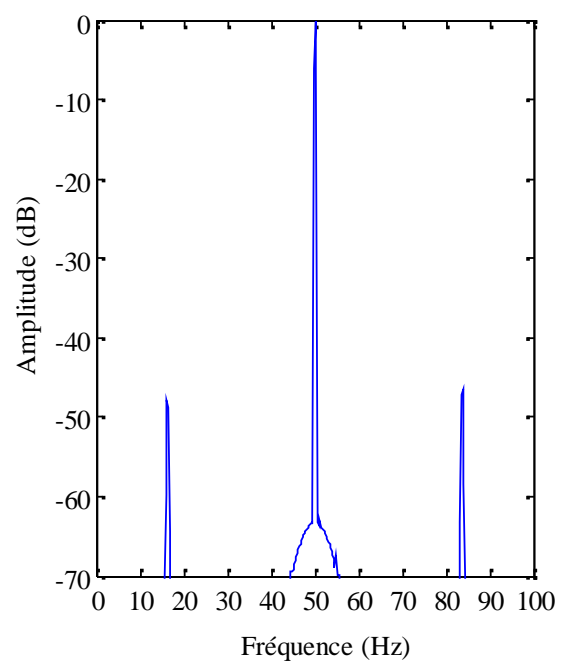

c) Courant statorique avec $10 \%$ de défaut

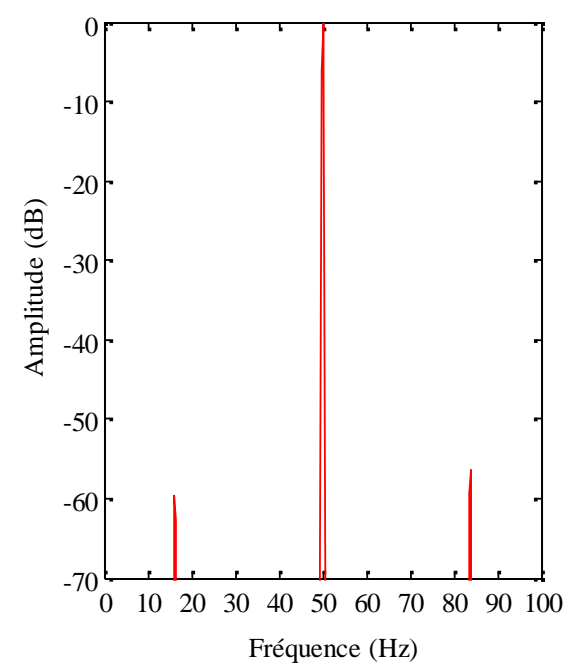

a') Induction magnétique à l'état sain

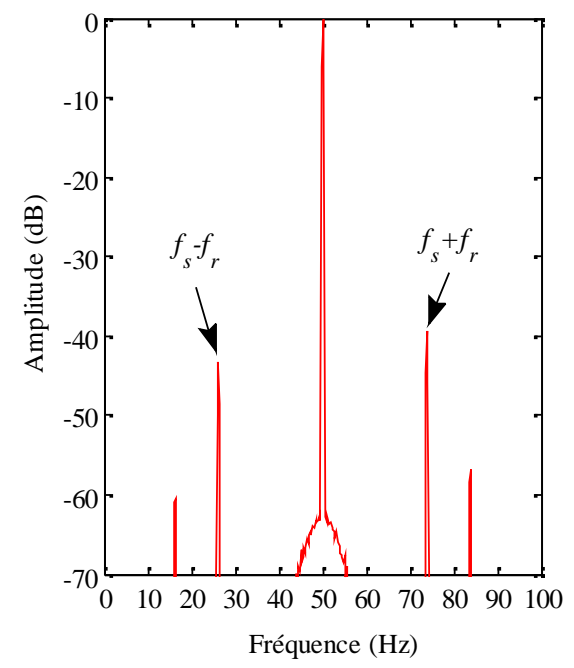

b') Induction magnétique avec $05 \%$ de défaut

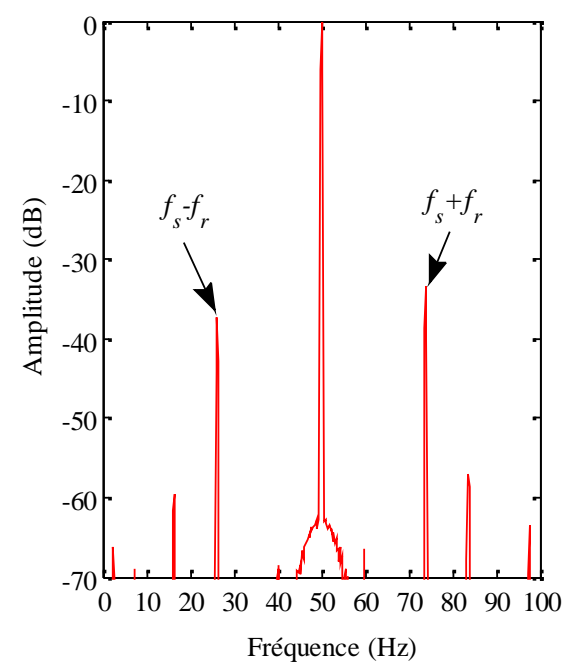

c') Induction magnétique avec $10 \%$ de défaut

Fig.9. Spectres du courant statorique et de l'induction magnétique à l'extérieur du moteur à l'état sain et avec défaut d'excentricité statique (en pleine charge) 


\section{CONCLUSION}

Au terme de ce travail nous pouvons signaler des pistes d'investigations pour parfaire les résultats obtenus. Les arguments quant à l'inefficacité de la méthode d'analyse spectrale du courant statorique semblent probants en matière de détection des index d'excentricité statique seule.

Le recours à la méthode d'analyse spectrale de la densité du flux magnétique à l'extérieur du moteur est à promouvoir avec des indicateurs d'efficacité validés même pour des faibles pourcentages de défaut, dans cette étude nous avons détecté avec certitude les signatures spectrales de défaut excentricité statique dans un moteur à induction renfermant les deux PSH ce qui constitue l'objectif premier de ce travail.

Les tests réalisés dans ce contexte confirment le bien fondé de notre choix technique. L'utilisation de cette méthode à été rendu possible par la modélisation de la méthode des éléments finis basée sur les circuits couplés; l'effet d'encochage et la saturation magnétique sont pris en compte. Ces éléments sont obligatoires pour l'étude de défauts d'excentricité. Ce choix n'est plus nécessaire pour estimer d'autre type de défauts (cassure de barre, etc ...).

\section{REMERCIEMENTS}

Les auteurs adressent leurs plus vifs remerciements au Professeur Champenois du laboratoire LAII Poitiers France pour son aimable contribution.

\section{RÉFÉRENCES}

[1] Arkkio A. Unbalanced Magnetic Pull in a High Speed Induction Motor with an Eccentric Rotor. IET Conference Publications, International Conference on Electrical Machines and Drives, 1997, 36-40.

[2] Sinervo A and Arkkio A. Eccentricity Related Forces in Two-Pole Induction Motor with Four-Pole Stator Damper Winding Analyzed Using Measured Rotor Orbits. IEEE Transactions on Magnetics, 2013, 49(6), 3029-3037. 
[3] Tenhunen A. Finite-Element Calculation of Unbalanced Magnetic Pull and Circulating Current between Parallel Windings in Induction Motor with Non-Uniform Eccentric Rotor. Proceedings of Electromotion'01, 2001, 19-24.

[4] Xiaodong L, Qing W and Nandi S. Performance Analysis of Three-Phase Induction Machine with Inclined Static Eccentricity. IEEE Transactions on Industry Applications, 2007, 43(2), 531-541.

[5] Dorrell D. G. Sources and Characteristics of Unbalanced Magnetic Pull in Three-Phase Cage Induction Motor with Axial-Varying Rotor Eccentricity. IEEE Transactions on Industry Applications, 2011, 47(1), 12-24.

[6] Nandi S, Bharadwaj R. M and Toliyat H. A. Performance Analyse of Three-Phase Induction Motor under Mixed Eccentricity Condition. IEEE Transactions on Energy Conversion, 2002, 17(3), 392-399.

[7] Nandi S, Bharadwaj R. M and Toliyat H. A. Mixed Eccentricity in Three Phase Induction Machines: Analysis, Simulation and Experiments. Conference Record of the Industry Applications Conference, $37^{\text {th }}$ IAS Annual Meeting, 2002, 3, 1525-1532.

[8] Nandi S, Toliyat H. A and Li X. Condition Monitoring and Fault Diagnosis of Electrical Motors-A Review. IEEE Transactions on Energy Conversion, 2005, 20(4), 719-729.

[9] Nandi S, Ilamparithi T. C, Lee S. B and Hyun D. Detection of Eccentricity Faults in Induction Machines Based on Nameplate Parameters. IEEE Transactions on Industrial Electronics, 2011, 58(1), 1673-1683.

[10] Nandi S. A Detailed Model of Induction Machines with Saturation Extendable for Fault Analysis. IEEE Transactions on Industry Applications, 2004, 40(5), 1302-1309.

[11] Benbouzid M. E. H, Vieira M and Theys C. Induction Motors' Faults Detection and Localization using Stator Current Advanced Signal Processing Techniques. IEEE Transactions on Power Electronics, 1999, 14(1), 14-22.

[12] Dorrell D. G, Thomson W. T and Roach S. Analysis of Airgap Flux, Current, and Vibration Signals as a function of the combination of Static and Dynamic Airgap Eccentricity in 3-Phase Induction Motors. IEEE Transactions on Industry Applications, 1997, 33(1), 24-34. 
[13] Thomson W. T and Fenger M. Current Signature Analysis to Detect Induction Motor Faults. IEEE Industry Applications Magazine, 2001, 7(4), 26-34.

[14] Gyftakis K. N, Spyropoulos D. V, Kappatou J. C and Mitronikas E. D. A Novel Approach for Broken Bar Fault Diagnosis in Induction Motors Through Torque Monitoring. IEEE Transactions on Energy Conversion, 2013, 28(2), 267-277.

[15] Halem N, Zouzou S. E, Srairi K, Guedidi S and Abbood F. A. Static Eccentricity Fault Diagnosis using the Signatures Analysis of Stator Current and Air Gap Magnetic Flux by Finite Element Method in Saturated Induction Motors. International Journal of System Assurance Engineering and Management, Springer Verlag, 2013, 4(2), 118-128.

[16] Halem N, Zouzou S. E, Srairi K, Guedidi S and Abbood F. A. Detection of Static Eccentricity Fault in Saturated Induction Motors by Air-Gap Magnetic Flux Signature Analysis using Finite Element Method. Journal of Fundamental and Applied Sciences, 2013, 5(1), 51-66.

[17] Ceban A, Pusca R and Romary R. Study of Rotor Faults in Induction Motors using External Magnetic Field Analysis. IEEE Transactions on Industrial Electronics, 2012, 59(5), 2082-2093.

[18] Vitek O, Janda M and Hajek V. Effects of Eccentricity on External Magnetic Field of Induction Machine. IEEE Conference Publication, $15^{\text {th }}$ IEEE Mediterranean Electro-technical Conference, 2010, 939-943.

[19] Kia S. H, Henao H, Capolino G. H and Martis C. Induction Machine Broken Bars Fault Detection using Stray Flux After Supply Disconnection. IEEE Conference Publication, $32^{\text {nd }}$ Annual Conference on Industrial Electronics, 2006, 1498-1503.

[20] Henao H, Demian C and Capolino G. A. A Frequency-Domain Detection of Stator Winding Faults in Induction Machines using an External Flux Sensor. IEEE Transactions on Industry Applications, 2003, 39(5), 1272-1279.

[21] Belkhayat D, Romary R, El Adnani M, Corton R and Brudny J. F. Fault Diagnosis in Induction Motors using Radial Field Measurement with an Antenna. IOPSCIENCE, Journal of Measurment Science and Technology, 2003, 14(9), 1695-1700. 
[22] Salah M, Bacha K and Chaari A. An Improved Spectral Analysis of the Stray Flux Component for the Detection of Air-Gap Irregularities in Squirrel Cage Motors. ISA Transactions, 2014, 53(3), 816-826

[23] Reece A. B. J and Preston T. W. Finite Element Methods in Electrical Power Engineering. $1^{\text {st }}$ Edition, Oxford Science Publications, Oxford University Press, NISBN 019856504 6, 2000.

[24] Joksimovic G. Line Current Spectrum Analysis in Saturated Three-Phase Cage Induction Machine. Electrical Engineering, Springer Verlag, 2010, 91(8), 425-437.

[25] Joksimovic G, Riger J, Wolbank T. M, Peric N and Vasak M. Stator-Current Spectrum Signature of Healthy Cage Rotor Induction Machines. IEEE Transactions on Industrial Electronics, 2013, 60(9), 4025-4033.

[26] Ferrah A, Hogben-Laing P. J, Bradley K. J, Asher G. M and Woolfson M. S. The Effect of Rotor Design of Sensorless Speed Estimation Using Rotor Slot Harmonics Identified by Adaptive Digital Filtering using the Maximum Likelihood Approach. Industry Applications Conference, Thirty-Second IAS Annual Meeting, IAS '97, Conference Record of the 1997 IEEE, 128-135.

[27] Nandi S, Ahmed S and Toliyat H. Detection of Rotor Slot and other Eccentricity Related Harmonics in a Three Phase Induction Motor with Different Rotor Cages. IEEE Transactions on Energy Conversion, 2001, 16(3), 253-260.

\section{How to cite this article:}

Halem N, Zouzou S. E and Ghodbane H. Detection of static eccentricity fault in PSH induction motor by using external magnetic flux density. J. Fundam. Appl. Sci., 2016, 8(3), 839-855. 